\title{
Suppressor of Cytokine Signaling 3: Emerging Role Linking Central Insulin Resistance and Alzheimer's Disease
}

\author{
Lan $\mathrm{Cao}^{1}$, Zigao Wang ${ }^{2 *}$ and Wenbin Wan ${ }^{3 *}$ \\ ${ }^{1}$ The State Key Laboratory of Medical Neurobiology, The Institutes of Brain Science and the Collaborative Innovation Center \\ for Brain Science, Shanghai Medical College, Fudan University, Shanghai, China, ${ }^{2}$ Department of Neurology, Huashan \\ Hospital, Fudan University, Shanghai, China, ${ }^{3}$ Department of Neurology, Renji Hospital, School of Medicine, Shanghai Jiao \\ Tong University, Shanghai, China
}

Currently, the etiology of Alzheimer's disease (AD) is still elusive. Central insulin resistance has been determined to play an important role in the progress of AD. However, the mechanism underlying the development of disrupted insulin signaling pathways in AD is

OPEN ACCESS

Edited by:

Umberto di Porzio,

Consiglio Nazionale delle Ricerche

(CNR), Italy

Reviewed by:

Cláudia Guimas Almeida,

Universidade Nova de Lisboa,

Portugal

Xuemin Xu,

University of Texas of the Permian

Basin, United States

*Correspondence:

Zigao Wang

zigaowang@163.com

Wenbin Wan

doctorwwb@163.com

Specialty section:

This article was submitted to

Neurodegeneration,

a section of the journal

Frontiers in Neuroscience

Received: 22 October 2017

Accepted: 01 June 2018

Published: 20 June 2018

Citation:

Cao L, Wang Z and Wan W (2018) Suppressor of Cytokine Signaling 3: Emerging Role Linking Central Insulin

Resistance and Alzheimer's Disease.

Front. Neurosci. 12:417.

doi: 10.3389/fnins.2018.00417 unclear. Suppressor of cytokine signaling 3 (SOCS3) is a member of the SOCS protein family that acts as a negative modulator of insulin signaling in sensitive tissues, such as hepatocytes and adipocytes. However, little is known about its role in neurological diseases. Recent evidence indicates that the level of SOCS3 is increased in the brains of individuals with $A D$, especially in areas with amyloid beta deposition, suggesting that SOCS3 may regulate the central insulin signaling pathways in AD. Here, we discuss the potential role of SOCS3 in AD and speculate that SOCS3 may be a promising therapeutic target for the treatment of $A D$.

Keywords: Alzheimer's disease, insulin resistance, socs3, neuroinflammation, therapeutic target

\section{INTRODUCTION}

Alzheimer's disease (AD) is a devastating neurodegenerative disease that leads to dementia. It is clinically characterized by the progressive loss of learning and memory and by abnormal mental states and behaviors; pathologically, it is characterized by extracellular amyloid beta $(A \beta)$ deposition and intracellular hyperphosphorylation of tau protein in the brain (Wan et al., 2015, 2016). Extensive efforts have been made to understand the underlying mechanisms of this disease. However, the etiology of $\mathrm{AD}$ is still elusive, and we still have no truly effective therapeutic agents to prevent the progression of the disease.

Insulin signaling is activated by circulating insulin and promotes a variety of metabolic pathways, including glucose storage and uptake, protein and lipid synthesis, and mitogenic responses (Chang et al., 2004). Insulin signaling is also critical for cell survival and for the maintenance of physiological functions in multiple tissues (Chang et al., 2004). Thus, disrupted insulin signaling - the most common of which is insulin resistance-can give rise to dysfunctions in both peripheral organs and the central nervous system (CNS). Insulin resistance is a pathological condition involving a failed response to normal levels of insulin. It is a precursor to diabetes mellitus as well as a noteworthy determinant in other metabolic disorders (Soumaya, 2012; Gustafson et al., 2015; Mayans, 2015). Insulin signaling in the CNS is important because of 
the conspicuous relationship between dysfunctional insulin signaling and disturbed learning and memory in neurodegenerative diseases, such as AD (van der Heide et al., 2006; Chen et al., 2014; Cai et al., 2015). Growing evidence suggests that insulin signaling may be a promising therapeutic target for the treatment of $\mathrm{AD}$. Insulin treatment by intranasal administration has consistently been shown to decrease the production of $A \beta$ and to ameliorate learning and memory deficits in individuals with AD (Reger et al., 2008a,b; Craft et al., 2012; Claxton et al., 2015). However, the molecular mechanisms responsible for the generation of insulin resistance in $\mathrm{AD}$ are still unclear.

Suppressor of cytokine signaling 3 (SOCS3) is a member of the SOCS protein family, which was initially characterized by its members' negative regulatory effects on cytokine signaling, including insulin signaling, in adipocytes (Emanuelli et al., 2000). However, the role of SOCS proteins in the CNS remains unclear. Recently, investigators have reported increased levels of SOCS3 in the brain tissues of individuals with AD (Walker et al., 2015; Cianciulli et al., 2017). Notably, the levels of SOCS3 are correlated with the deposition of A $\beta$ (Walker et al., 2015; Cianciulli et al., 2017; Iwahara et al., 2017), suggesting that SOCS3 might be a novel target that regulates insulin signaling in the brains of $\mathrm{AD}$ patients.

In the present review, we first discuss evidence from published studies that have examined the potential role of SOCS3 in AD, suggesting that SOCS3 is a potential target in the treatment of $\mathrm{AD}$.

\section{INSULIN RESISTANCE IN AD}

Insulin recognizes the $\alpha$-subunit of the insulin receptor, subsequently leading to autophosphorylation of tyrosine residues and activation of the intracellular $\beta$-subunit (IR $\beta$ ) (Lee and Pilch, 1994). Insulin receptor substrates (IRS) are then tyrosinephosphorylated, and the downstream insulin signaling pathway is activated (Lee and Pilch, 1994). During a state of insulin resistance, cells exhibit decreased sensitivity to stimulation by insulin, which presents as a reduction in the tyrosine phosphorylation levels of IR $\beta$ and IRS and results in insufficient uptake of glucose and amino acids (Boucher et al., 2014). Insulin resistance in neurons also results in many other disturbances, such as synapse dysfunction, disruption of dendritic spines, and disorders of neurotransmitter metabolism (Kleinridders et al., 2014).

Accumulating evidence indicates that insulin resistance plays a critical role in the development of AD (Frame and Zheleva, 2006; Liu et al., 2011; Morris and Burns, 2012; Son et al., 2012; Talbot et al., 2012; Chen and Zhong, 2013; Cai et al., 2015; Mullins et al., 2017). Insulin signaling regulates amyloid precursor protein (APP) metabolism through $\alpha-, \beta$-, and $\gamma$-secretases. During insulin resistance, the enzyme activities of $\beta$ - and $\gamma$-secretase increase, which subsequently leads to the overproduction of $A \beta$ in neurons (Son et al., 2012). Furthermore, accumulation of $A \beta$ may also enhance the degree of insulin resistance and amplify the damage to the brain (Cai et al., 2015). Aberrant phosphorylation of the tau protein in neurons is another important hallmark of AD. Interestingly, the tau phosphorylation levels are also greatly modulated by insulin signaling (Mullins et al., 2017). In rodent models of insulin resistance, abundant phosphorylation of tau has been identified. More importantly, insulin treatment is able to ameliorate the tau pathology; the activity of glycogen synthase kinase-3 (GSK-3) is considered essential for this effect (Frame and Zheleva, 2006; Chen and Zhong, 2013).

In $\mathrm{AD}$ individuals, brain insulin signaling is disturbed, as has been determined from postmortem tissues (Liu et al., 2011; Morris and Burns, 2012). Specifically, the protein levels of the insulin receptor and its substrates are decreased compared to the levels in the brains of individuals without $\mathrm{AD}$ (Liu et al., 2011; Morris and Burns, 2012). Talbot and co-workers demonstrated that cultured brain slices from $\mathrm{AD}$ patients were insensitive to insulin treatment, revealing the presence of insulin resistance in $\mathrm{AD}$ brains (Talbot et al., 2012). Thus, targeting insulin signaling pathways in the brain is a promising therapy for $\mathrm{AD}$. In previous studies, insulin has been employed to treat individuals with AD. These studies found that intranasal insulin treatment delayed memory loss, improved patients' cognition, and ameliorated the accumulation of plaques in the brain (Reger et al., 2008b; Dhamoon et al., 2009; Stein et al., 2011; Craft et al., 2012). An insulin-signaling sensitizing agent, metformin, has been highlighted as a potential therapeutic agent for $\mathrm{AD}$ because of its protective effects against memory impairment and $A \beta$ deposition (Ou et al., 2017). In that study, researchers also found that metformin triggered neurogenesis and presented anti-inflammatory activity in the brains of $\mathrm{AD}$ mice $(\mathrm{Ou}$ et al., 2017). Although these findings indicate that administration of insulin may be helpful in treating $\mathrm{AD}$, the effectiveness and safety of this treatment require further investigation. Furthermore, contrary evidence still exists, as metformin has also been found to facilitate the generation of $\mathrm{A} \beta$ via upregulation of $\beta$ - and $\gamma$-secretases (Chen et al., 2009; Son et al., 2016); however, in these studies, a familial disease model of $\mathrm{AD}$ was employed, although sporadic $\mathrm{AD}$ is the most common type of the disease. Thus, overall, the details regarding the development of dysfunctional insulin signaling in the brain are presently unclear, and the underlying mechanisms of insulin resistance in $\mathrm{AD}$ require further elaboration.

\section{SOCS3 AS A NEGATIVE REGULATOR IN INSULIN SIGNALING}

The protein members of the SOCS family were first identified in 1997 and have been determined to act as negative modulators of cytokine signaling in various tissues, such as adipocytes, hepatocytes, and tissues of the immune system and CNS (Endo et al., 1997; Naka et al., 1997; Starr et al., 1997). Thus far, eight structurally similar members, including SOCS1-7 and the cytokine-inducible Src homology 2-containing (SH2) protein (CIS), have been characterized in this family (Howard and Flier, 2006). The SOCS proteins comprise three distinct domains: an $N$-terminal domain with a variable length but without a recognizable motif, a well conserved central $\mathrm{SH} 2$ domain that 
binds tyrosine phosphorylation, and a more highly conserved C-terminal domain of $\sim 40$ amino acids known as the "SOCS box" (Bullock et al., 2007). Expression of SOCS proteins is mainly increased by the activation of the signal transducer and activator of transcription (STAT) signaling pathway and partially by the NF- $\kappa \mathrm{B}$ pathway, both of which are induced by stimuli that interact with their receptors (Rui et al., 2002; Lebrun and Van Obberghen, 2008). By contacting target elements, such as Janus activated kinase (JAK) or tyrosine-phosphorylated cytokine receptors, SOCS proteins inhibit the progress of phosphorylation and subsequently prevent the activation of STAT-induced transcription factors, thus resulting in negative feedback (Lebrun and Van Obberghen, 2008). Expression of SOCS genes is normally extremely low but increases after exposure to cytokines and hormones, such as lipopolysaccharide (LPS) and insulin (Kazi et al., 2014).

The specificity of each member of the SOCS family is not clear. Previously reviews have concisely reported the characteristic of each one in SCOS (Kile and Alexander, 2001; Larsen and Ropke, 2002; Linossi et al., 2013; Kazi et al., 2014). However, SOCS3 is instrumental, as its kinase inhibitory region (KIR) in the $N$-terminal domain is next to the SH2 domain (Lebrun and Van Obberghen, 2008). The KIR is a functional region that inhibits the kinase activity of JAK and insulin receptors (Lebrun and Van Obberghen, 2008). Very recently, two other recognizable regions, the extended $\mathrm{SH} 2$ subdomain (ESS; located between the KIR and $\mathrm{SH} 2$ domains) and proline (P)-glutamic acid (E)-serine (S)-threonine (T) (PEST) motif (within the SH2 region), have been identified in SOCS3 (Babon et al., 2006; Lebrun and Van Obberghen, 2008). The ESS is a crucial domain that interacts directly with the nearby tyrosine phosphorylation binding site (Babon et al., 2006). The PEST motif has an important intracellular role in promoting SOCS3 turnover and affecting its degradation pathway (Babon et al., 2006). The functions of these domains suggest that the expression of SOCS3 is finely regulated.

Accumulating evidence has identified SOCS3 as an important negative regulator of the insulin signaling pathway in major insulin-sensitive tissues, such as hepatocytes and adipocytes (Rui et al., 2002; Howard and Flier, 2006; Yang et al., 2010). Insulin stimulation results in the upregulation of SOCS3, which subsequently blocks the insulin signaling pathway (Rui et al., 2002; Howard and Flier, 2006; Yang et al., 2010), suggesting that SOCS3 plays an essential role in regulating insulin sensitivity. Recently, clinical investigations have revealed that individuals with insulin resistance, or with a high risk of insulin resistance, have increased SOCS3 levels (Ghanim et al., 2007, 2009). In genetic analyses, SOCS3 polymorphisms and epigenetic methylation have also been associated with insulin resistance (Ali et al., 2016; Boyraz et al., 2016). Based on these findings, it is reasonable to speculate that SOCS3 is involved in the development of insulin resistance in the human body and that increased levels of SOCS3 could lead to pathological conditions that disrupt the insulin signaling pathway.

In rodent models of insulin resistance, SOCS3 mRNA expression is robustly upregulated in almost all insulin-sensitive tissues, including hepatic, skeletal, and adipose tissues (Emanuelli et al., 2001; Ueki et al., 2004a,b), revealing a strong and direct relationship between SOCS and insulin resistance. Interestingly, no change in SOCS3 expression is observed in rodent models with a deficiency in TNF $\alpha$ receptors (Uysal et al., 1997), indicating that the expression of SOCS3 is dependent on an inflammatory $\mathrm{TNF} \alpha$ signal. Several studies have examined the genetic regulation of SOCS3 in different peripheral organs to determine its role in the generation of insulin resistance; these studies have shown that SOCS3 deletion results in the loss of insulin resistance and in enhanced effects of insulin signaling, as evidenced by increased tyrosine phosphorylation of IRS1 (Torisu et al., 2007; Sachithanandan et al., 2010; Jorgensen et al., 2013). Consistent with such findings, SOCS3 overexpression decreases the tyrosine phosphorylation levels of IRS1 and inhibits the activity of phosphatidylinositol-3 kinase (PI3K), a downstream signaling element of IRS1 (Ueki et al., 2004a; Yang et al., 2012), revealing a pivotal role of SOCS3 in insulin resistance. Furthermore, SOCS3-induced ubiquitin-mediated degradation of IRS1 has been demonstrated to participate in the inhibition of insulin in multiple cell types (Rui et al., 2002). Specifically, SOCS3 binds with IRS1 and promotes its ubiquitination (Rui et al., 2002). An elongin BCcontaining ubiquitin ligase is subsequently incorporated, which finally results in the degradation of the IRS1 protein (Rui et al., 2002).

Thus, SOCS3 is critical for the inhibition of the insulin signaling pathway. The aforementioned investigations indicate that suppression of SOCS3 is beneficial for the activation of insulin signaling and suggest that SOCS3 could be a new therapeutic target for diseases in which insulin resistance is involved.

\section{SOCS3 IN THE BRAIN}

The expression of SOCS3 in the brain was first discovered in the year 2000; detection of mRNA revealed that SOCS3 is apparently widespread in the brain, including in the hippocampus, the granular layer of the cerebellum, the thalamus and the basal ganglia (Polizzotto et al., 2000; Wang and Campbell, 2002). Under physiological conditions, the SOCS3 transcript achieves its maximal levels in the brain from E14 to postnatal day eight, but it drops to quite a low level in adults (Polizzotto et al., 2000). Furthermore, Mishra et al. reported that upregulation of SOCS3 promoted the differentiation of cultured neural stem cells even if no NGF was added to the medium, indicating that SOCS3 is involved in the development of the CNS.

As in peripheral tissues, expression of SOCS3 in the CNS increases rapidly in response to various stress-related stimuli, such as LPS, IFN, IL1, and IL6 (Wang and Campbell, 2002; Steffensen et al., 2014). Qin and coworkers found that IFN $\beta$-induced expression of SOCS3 in astrocytes was dependent on the activation of STAT3. Disruption of SOCS3 by the administration of IFN $\beta$ resulted in a large mass of inflammatory cytokines and enhanced the migration of microglial and $\mathrm{T}$ cells (Qin et al., 2008). These results indicate that SOCS3 could act as an immune modulator in the CNS. 
The precise roles of SOCS3 in the mature neurons of the CNS are still unclear. Nevertheless, it has been found that SOCS3 acts as a negative regulator of neuronal survival and axon regeneration after neural injury (Smith et al., 2009; Liu et al., 2015). Deletion of SOCS3 facilitates cellular survival and axon regeneration in retinal ganglion cells after optic nerve injury (Smith et al., 2009; Liu et al., 2015). Thus, SOCS3 could be a promising modulator of neural repair in the adult CNS, perhaps guarding against long-distance axon regeneration.

\section{SOCS3 IN AD}

Expression of SOCS3 has been well defined in human brains, but changes in SOCS3 expression in AD brains had not been examined until a study by Walker and co-workers (Walker et al., 2015). In this work, the researchers found that expression of SOCS3 in the brains of AD patients was significantly greater than expression in the brains of individuals with mild cognitive impairment (MCI) or the brains of non-demented individuals (Walker et al., 2015). They also observed significant correlations between the SOCS3 mRNA levels and scores for A $\beta$ plaques and neurofibrillary tangles (Walker et al., 2015). In alignment with SOCS3's aforementioned involvement in CNS inflammation, the level of SOCS3 is consistently increased in $A \beta$-stimulated human microglia (Walker et al., 2015). Furthermore, expression of SOCS3 is regulated by the JAK/STAT signaling pathway (Lebrun and Van Obberghen, 2008). In fact, the JAK/STAT signal transduction pathway can be activated by exposure to $\mathrm{A} \beta$ (Capiralla et al., 2012), indicating a potential role for $\mathrm{A} \beta$ in regulating the expression of SOCS3. Recently, Iwahara and colleagues demonstrated that both $\mathrm{A} \beta$-stimulated primary cultured microglia and microglia in a APPswe/PS1dE9 transgenic mouse model of AD expressed SOCS3; they further revealed that SOCS3 was involved in the switch to an activated microglia phenotype (Iwahara et al., 2017), suggesting a potential role for SOCS3 in AD, especially in AD-related neuroinflammation.

However, the effect of SOCS3 on insulin resistance in AD has not been defined. Studies have shown that in addition to glucose metabolism and neural activation, impaired neuronal insulin signaling is also involved in proinflammatory signaling associated with AD (Ferreira et al., 2014). In postmortem human brains and in rodent models of $\mathrm{AD}, \mathrm{A} \beta$ accumulation has been shown to cause deficiencies in insulin signaling, whereby serine phosphorylation of IRS1 is increased and tyrosine phosphorylation of IRS1 is decreased (Bomfim et al., 2012). Indeed, tyrosine phosphorylation of IRS1 acts as an important proinflammatory signal (Bomfim et al., 2012; Ferreira et al., 2014). Subsequent research has revealed that the neurotoxicity resulting from $A \beta$ inhibits tyrosine phosphorylation-mediated signaling by IRS1 and leads to serine phosphorylation-induced activation of IRS1 via TNFa (Ma et al., 2009; Bomfim et al., 2012; Craft, 2012; Ferreira et al., 2014), a modulator of SOCS3, as described above (Uysal et al., 1997).

Taken together, these studies suggest that SOCS3 is involved in dysfunctional insulin signaling in the brains of patients with $\mathrm{AD}$ (Figure 1). As SOCS3 has also been identified in peripheral tissues, we speculate that SOCS3 plays an essential role in the establishment of insulin resistance in AD. Further study is warranted to determine the specific relationship between SOCS3 and insulin resistance.

\section{SOCS3-MODULATED INFLAMMATORY CYTOKINES IN AD}

As it has been proposed that insulin resistance in diabetes is a consequent result of chronic inflammation leading to the overloaded proinflammatory cytokines (Vieira et al., 2017). The inflammatory mediators are able to cross the blood-brain barrier $(\mathrm{BBB})$ and activate the microglia. Growing evidence has revealed the existence of inflammation in $\mathrm{AD}$ brain and peripheral blood (Czirr and Wyss-Coray, 2012; Clarke et al., 2015). It also shows neuroinflammation regulates the impairment of cognition (Heneka and O'Banion, 2007), suggesting that inflammation acts as a linkage between insulin resistance in diabetic and in AD.

In peripheral, activation of inflammatory signal promotes the expression of SOCS3 (Uysal et al., 1997; Yin et al., 2015). In CNS, accumulating evidence revealed the regulatory role of SOCS3 in neuroinflammation. Microglia is the resident immune cells in the brain. Microglia-associated central inflammation plays a deleterious role in neural degeneration resulting in the pathogenesis of AD. Recently, Iwahara and co-workers observed that microglia expressed SOCS3 and exhibited an M1-like phenotype, expression cytokine TNF $\alpha$ but not IL6 in A $\beta$-stimulated primary culture and APP/PS1 mice (Iwahara et al., 2017). While, elimination of SOCS3 expression resulted in upregulation of IL6 in A $\beta$-challenged primary microglia (Iwahara et al., 2017), suggesting the role of SOCS3 in suppressing the excessive inflammation induced by M1 microglia. However, the effect of SOCS3 in the rodent AD mice has not been evaluated in this work.

Yet in neuron, the role of SOCS3 presents very different from that in microglia. SOCS3-overexpression inhibited the JAK/STAT3-regulated protective effects of IGF1 against $\mathrm{TNF} \alpha$-induced lesions and led to neuroblastoma cell death, indicating the involvement of SOCS3 in cell survival (Yadav et al., 2005). In primary-cultured sensory neurons, SOCS3 suppressed neurite growth via the inhibition of STAT3 signal, suggesting the detrimental effect of SOCS3 on axonal growth (Miao et al., 2006).

\section{THE ROLE OF SOCS3 IN AD}

Although many studies have been conducted to explore therapeutic candidates for the prevention and treatment of $\mathrm{AD}$, an effective target has yet to be determined. Previous work has revealed that the $A \beta$ and tau proteins are the two core pathologies in AD brains (Cowan and Mudher, 2013; Wan et al., 2014; Villemagne et al., 2015; Xu et al., 2015). However, in past few decades, multiple treatments, including $A \beta$ immunization (Holmes et al., 2008; Doody et al., 2013) and administration of $\beta$-secretase inhibitors (Mikulca et al., 2014), $\gamma$-secretase inhibitors (Mikulca et al., 2014; Doody et al., 2016), and tau 

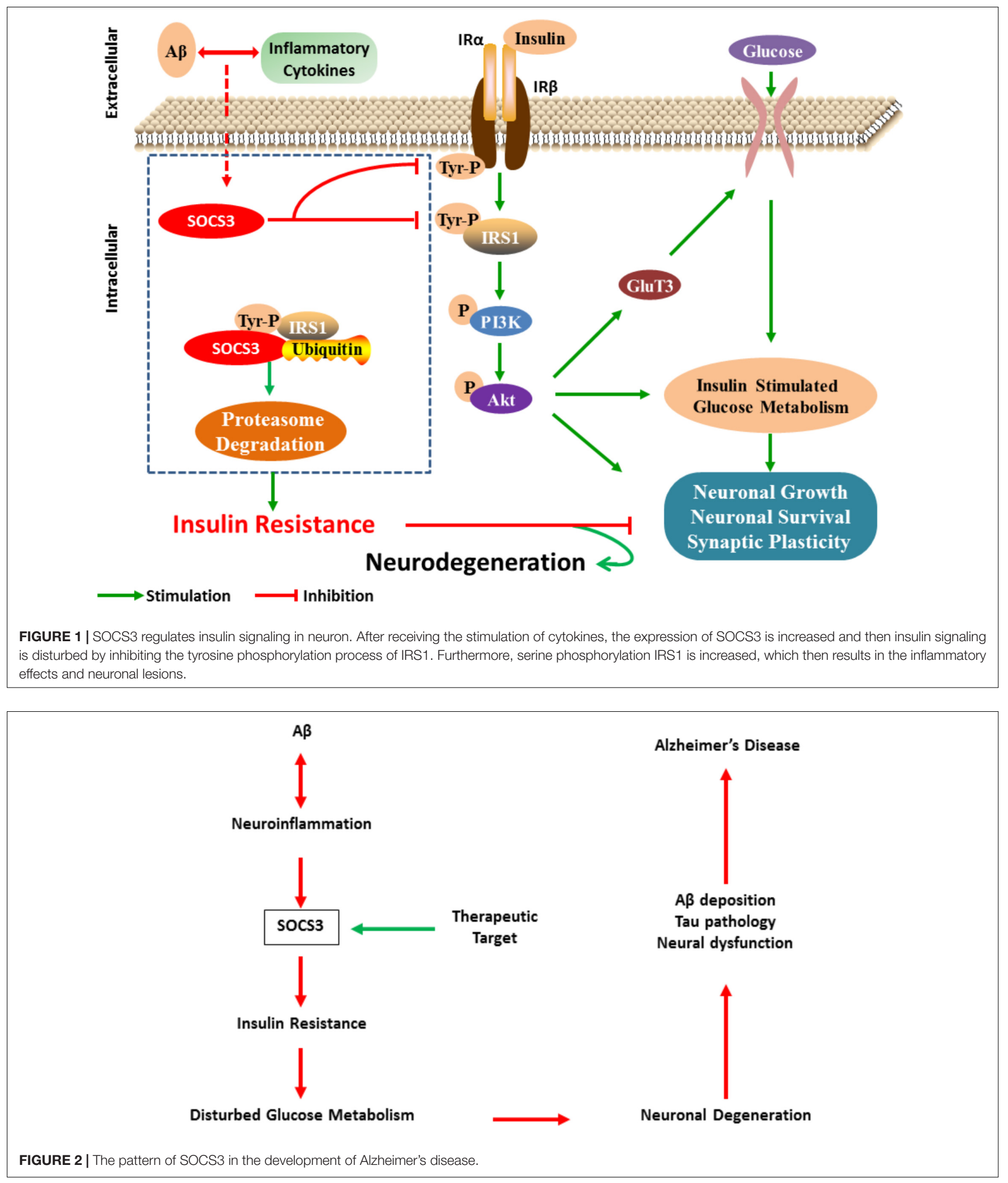

aggregation inhibitors (Gauthier et al., 2016), have proven to be insufficient to cure $\mathrm{AD}$. Therefore, it is imperative to find more promising targets for disease prevention.
Dysfunctional cerebral glucose metabolism is an early and invariant characteristic of AD (Chen and Zhong, 2013). Some research suggests that dysfunctional neuron metabolism is a 
critical contributor to AD (Chen and Zhong, 2013). FDG$\mathrm{PET} / \mathrm{CT}$ imaging shows consistent and progressive reductions in brain glucose metabolism in individuals with $\mathrm{AD}$, and these reductions are closely correlated with cognitive impairment and clinical severity (Mosconi, 2005). Since insulin signaling is the key factor regulating glycometabolism, it is important to analyse disrupted insulin signaling pathways in $\mathrm{AD}$ and to determine the underlying pathogenesis.

As a negative modulator, SOCS3 is a vital switch for the unhindered transduction of insulin signals, which subsequently affects cellular glycometabolism. In peripheral tissues, inhibition of SOCS3 has been proven to activate insulin signaling (Torisu et al., 2007; Sachithanandan et al., 2010; Jorgensen et al., 2013), and expression of SOCS3 is increased in the brains of individuals with AD (Walker et al., 2015; Iwahara et al., 2017). Thus, SOCS3 may also be a promising candidate for ameliorating insulin insensitivity, leading to the improvement of brain glycometabolism and the prevention of disease progression.

Although direct evidence is still insufficient, relevant studies have revealed that SOCS3 may be a promising target for the treatment of AD (Czirr and Wyss-Coray, 2012; Clarke et al., 2015). SOCS3 is also involved in neroninflammation and is essential for inflammation-regulated insulin resistance in the central (Figure 2). Therefore, the accurate role of SOCS3 in AD is still unclear, but its detrimental effects on cell survival and axonal growth indicate SOCS3 as a potential target in neuroral protection (Yadav et al., 2005; Miao et al., 2006).

\section{CONCLUSION AND PERSPECTIVE}

The regulatory mechanisms of SOCS3 expression in AD are elusive. $\mathrm{A} \beta$ deposition and inflammatory factors in the brain are the most important causes of neural lesions (Zhang and Jiang,

\section{REFERENCES}

Ali, O., Cerjak, D., Kent, J. W. Jr., and James, R. (2016). Methylation of SOCS3 is inversely associated with metabolic syndrome in an epigenome-wide association study of obesity. Epigenetics 11, 699-707. doi: 10.1080/15592294. 2016.1216284

Babon, J. J., Mcmanus, E. J., Yao, S., and Desouza, D. P. (2006). The structure of SOCS3 reveals the basis of the extended sh2 domain function and identifies an unstructured insertion that regulates stability. Mol. Cell 22, 205-216. doi: 10.1016/j.molcel.2006.03.024

Bomfim, T. R., Forny-Germano, L., Sathler, L. B., and Brito-Moreira, J. (2012). An anti-diabetes agent protects the mouse brain from defective insulin signaling caused by Alzheimer's disease- associated Abeta oligomers. J. Clin. Invest. 122, 1339-1353. doi: 10.1172/JCI57256

Boucher, J., Kleinridders, A., and Kahn, C. R. (2014). Insulin receptor signaling in normal and insulin-resistant states. Cold Spring Harb. Perspect. Biol 6:a009191. doi: 10.1101/cshperspect.a009191

Boyraz, M., Yesilkaya, E., Ezgu, F., and Bideci, A. (2016). Effect of cytokine signaling 3 gene polymorphisms in childhood obesity. J. Clin. Res. Pediatr. Endocrinol. 8, 452-460. doi: 10.4274/jcrpe.3167

Bullock, A. N., Rodriguez, M. C., Debreczeni, J. E., and Songyang, Z. (2007). Structure of the SOCS4-ElonginB/C complex reveals a distinct SOCS box interface and the molecular basis for SOCS-dependent EGFR degradation. Structure 15, 1493-1504. doi: 10.1016/j.str.2007. 09.016
2015) and may also initiate upregulation of SOCS3 expression in $\mathrm{AD}$. Cerebral glucose hypometabolism and insulin resistance are the invariant feature of $\mathrm{AD}$ and have been revealed to be critical for the development of the disease (Steen et al., 2005; Mosconi et al., 2008; Takeda et al., 2010). Insulin signaling is the most critical modulator of glucose metabolism in various tissues and organs. However, the mechanisms underlying the development of insulin resistance in brains of $\mathrm{AD}$ are still unclear. Current evidence as described above does not only indicate that SOCS3-mediated inhibition of insulin signaling is important for the peripheral pathologies in metabolic syndromes, but also suggest the essential role of SOCS3 in the disturbed insulin signaling in the CNS of $\mathrm{AD}$. We speculate that the increased level of SOCS3 resulting from the stimulations of pathological products in brains of $\mathrm{AD}$ might lead to the disruption of insulin signaling, which then facilitates the development of AD. Despite all these, further studies are needed to test our hypothesis and to inspect that targeting SOCS3 in the brain would be a promising therapeutic strategy for $\mathrm{AD}$.

\section{AUTHOR CONTRIBUTIONS}

LC and WW wrote this review. ZW corrected and made the revisions of this review.

\section{FUNDING}

This work was supported by the National Natural Science Foundation of China (Grant No. 81701056) and the Science and Technology Commission of Shanghai Municipality (Grant Nos. 20164Y0193 and 20174Y0168).

Cai, Z., Xiao, M., Chang, L., and Yan, L. J. (2015). Role of insulin resistance in Alzheimer's disease. Metab. Brain Dis. 30, 839-851. doi: 10.1007/s11011-0149631-3

Capiralla, H., Vingtdeux, V., Zhao, H., and Sankowski, R. (2012). Resveratrol mitigates lipopolysaccharide- and Abeta-mediated microglial inflammation by inhibiting the TLR4/NF-kappaB/STAT signaling cascade. J. Neurochem. 120, 461-472. doi: 10.1111/j.1471-4159.2011.07594.x

Chang, L., Chiang, S. H., and Saltiel, A. R. (2004). Insulin signaling and the regulation of glucose transport. Mol. Med. 10, 65-71.

Chen, Y., Deng, Y., Zhang, B., and Gong, C. X. (2014). Deregulation of brain insulin signaling in Alzheimer's disease. Neurosci. Bull. 30, 282-294. doi: 10.1007/s12264-013-1408-x

Chen, Y., Zhou, K., Wang, R., and Liu, Y. (2009). Antidiabetic drug metformin (GlucophageR) increases biogenesis of Alzheimer's amyloid peptides via upregulating BACE1 transcription. Proc. Natl. Acad. Sci. U.S.A. 106, 3907-3912. doi: 10.1073/pnas.0807991106

Chen, Z., and Zhong, C. (2013). Decoding Alzheimer's disease from perturbed cerebral glucose metabolism: implications for diagnostic and therapeutic strategies. Prog. Neurobiol. 108, 21-43. doi: 10.1016/j.pneurobio.2013.06.004

Cianciulli, A., Calvello, R., Porro, C., and Trotta, T. (2017). Understanding the role of SOCS signaling in neurodegenerative diseases: current and emerging concepts. Cytokine Growth Factor Rev. 37, 67-79. doi: 10.1016/j.cytogfr.2017. 07.005

Clarke, J. R., Lyra, E. S. N. M., Figueiredo, C. P., and Frozza, R. L. (2015). Alzheimer-associated Abeta oligomers impact the central nervous system 
to induce peripheral metabolic deregulation. EMBO Mol. Med. 7, 190-210. doi: 10.15252/emmm.201404183

Claxton, A., Baker, L. D., Hanson, A., and Trittschuh, E. H. (2015). Long-acting intranasal insulin detemir improves cognition for adults with mild cognitive impairment or early-stage Alzheimer's disease dementia. J. Alzheimers Dis. 44, 897-906. doi: 10.3233/JAD- 141791

Cowan, C. M., and Mudher, A. (2013). Are tau aggregates toxic or protective in tauopathies? Front. Neurol. 4:114. doi: 10.3389/fneur.2013.00114 doi: 10.3389/ fneur.2013.00114

Craft, S. (2012). Alzheimer disease: insulin resistance and AD-extending the translational path. Nat. Rev. Neurol. 8, 360-362. doi: 10.1038/nrneurol.20 12.112

Craft, S., Baker, L. D., Montine, T. J., and Minoshima, S. (2012). Intranasal insulin therapy for Alzheimer disease and amnestic mild cognitive impairment: a pilot clinical trial. Arch. Neurol. 69, 29-38. doi: 10.1001/archneurol.2011.233

Czirr, E., and Wyss-Coray, T. (2012). The immunology of neurodegeneration. J. Clin. Invest. 122, 1156-1163. doi: 10.1172/JCI58656

Dhamoon, M. S., Noble, J. M., and Craft, S. (2009). Intranasal insulin improves cognition and modulates beta-amyloid in early AD. Neurology 72, 292-293. doi: 10.1212/01.wnl.0000344246.91081.2c

Doody, R. S., Raman, R., Farlow, M., and Iwatsubo, T. (2013). A phase 3 trial of semagacestat for treatment of Alzheimer's disease. N. Engl. J. Med. 369, 341-350. doi: 10.1056/NEJMoa1210951

Doody, R. S., Raman, R., Fau-Farlow, M., Farlow, M., Fau-Iwatsubo, T., Iwatsubo, T., et al. (2016). A phase 3 trial of semagacestat for treatment of Alzheimer's disease. N. Engl. J. Med. 369, 341-350. doi: 10.1056/ NEJMoa1210951

Emanuelli, B., Peraldi, P., Filloux, C., and Chavey, C. (2001). SOCS-3 inhibits insulin signaling and is up-regulated in response to tumor necrosis factoralpha in the adipose tissue of obese mice. J. Biol. Chem. 276, 47944-47949. doi: 10.1074/jbc.M104602200

Emanuelli, B., Peraldi, P., Filloux, C., and Sawka-Verhelle, D. (2000). SOCS-3 is an insulin-induced negative regulator of insulin signaling. J. Biol. Chem. 275, 15985-15991. doi: 10.1074/jbc.275.21.15985

Endo, T. A., Masuhara, M., Yokouchi, M., and Suzuki, R. (1997). A new protein containing an SH2 domain that inhibits JAK kinases. Nature 387, 921-924. doi: $10.1038 / 43213$

Ferreira, S. T., Clarke, J. R., Bomfim, T. R., and De Felice, F. G. (2014). Inflammation, defective insulin signaling, and neuronal dysfunction in Alzheimer's disease. Alzheimers Dement. 10, S76-S83. doi: 10.1016/j.jalz.2013. 12.010

Frame, S., and Zheleva, D. (2006). Targeting glycogen synthase kinase- 3 in insulin signalling. Expert Opin. Ther. Targets 10, 429-444. doi: 10.1517/14728222.10.3. 429

Gauthier, S., Feldman, H. H., Schneider, L. S., and Wilcock, G. K. (2016). Efficacy and safety of tau-aggregation inhibitor therapy in patients with mild or moderate Alzheimer's disease: a randomised, controlled, double-blind, parallelarm, phase 3 trial. Lancet 388, 2873-2884. doi: 10.1016/S0140-6736(16)312752

Ghanim, H., Abuaysheh, S., Sia, C. L., and Korzeniewski, K. (2009). Increase in plasma endotoxin concentrations and the expression of Toll-like receptors and suppressor of cytokine signaling- 3 in mononuclear cells after a high-fat, high-carbohydrate meal: implications for insulin resistance. Diabetes Care 32, 2281-2287. doi: $10.2337 / \mathrm{dc} 09-0979$

Ghanim, H., Aljada, A., Daoud, N., and Deopurkar, R. (2007). Role of inflammatory mediators in the suppression of insulin receptor phosphorylation in circulating mononuclear cells of obese subjects. Diabetologia 50, 278-285. doi: 10.1007/ s00125-006-0508-9

Gustafson, B., Hedjazifar, S., Gogg, S., and Hammarstedt, A. (2015). Insulin resistance and impaired adipogenesis. Trends Endocrinol. Metab. 26, 193-200. doi: 10.1016/j.tem.2015.01.006

Heneka, M. T., and O'Banion, M. K. (2007). Inflammatory processes in Alzheimer's disease. J. Neuroimmunol. 184, 69-91. doi: 10.1016/j.jneuroim.2006. 11.017

Holmes, C., Boche, D., Wilkinson, D., and Yadegarfar, G. (2008). Long-term effects of Abeta42 immunisation in Alzheimer's disease: follow-up of a randomised, placebo-controlled phase I trial. Lancet 372, 216-223. doi: 10.1016/S01406736(08)61075-2
Howard, J. K., and Flier, J. S. (2006). Attenuation of leptin and insulin signaling by SOCS proteins. Trends Endocrinol. Metab. 17, 365-371. doi: 10.1016/j.tem. 2006.09.007

Iwahara, N., Hisahara, S., Kawamata, J., and Matsumura, A. (2017). Role of suppressor of cytokine signaling 3 (SOCS3) in altering activated microglia phenotype in APPswe/PS1dE9 Mice. J. Alzheimers Dis. 55, 1235-1247. doi: 10.3233/JAD-160887

Jorgensen, S. B., O'neill, H. M., Sylow, L., and Honeyman, J. (2013). Deletion of skeletal muscle SOCS3 prevents insulin resistance in obesity. Diabetes Metab. Res. Rev. 62, 56-64. doi: $10.2337 / \mathrm{db} 12-0443$

Kazi, J. U., Kabir, N. N., Flores-Morales, A., and Ronnstrand, L. (2014). SOCS proteins in regulation of receptor tyrosine kinase signaling. Cell Mol. Life Sci. 71, 3297-3310. doi: 10.1007/s00018-014-1619-y

Kile, B. T., and Alexander, W. S. (2001). The suppressors of cytokine signalling (SOCS). Cell Mol. Life. Sci. 58, 1627-1635. doi: 10.1007/PL00000801

Kleinridders, A., Ferris, H. A., Cai, W., and Kahn, C. R. (2014). Insulin action in brain regulates systemic metabolism and brain function. Diabetes Metab. Res. Rev. 63, 2232-2243. doi: 10.2337/db14-0568

Larsen, L., and Ropke, C. (2002). Suppressors of cytokine signalling: SOCS. APMIS 110, 833-844. doi: 10.1034/j.1600-0463.2002.1101201.x

Lebrun, P., and Van Obberghen, E. (2008). SOCS proteins causing trouble in insulin action. Acta Physiol. 192, 29-36. doi: 10.1111/j.1748-1716.2007.01 $782 . x$

Lee, J., and Pilch, P. F. (1994). The insulin receptor: structure, function, and signaling. Am J Physiol 266, C319-C334. doi: 10.1152/ajpcell.1994.266.2.C319

Linossi, E. M., Babon, J. J., Hilton, D. J., and Nicholson, S. E. (2013). Suppression of cytokine signaling: the SOCS perspective. Cytokine Growth Factor Rev. 24, 241-248. doi: 10.1016/j.cytogfr.2013.03.005

Liu, X., Williams, P. R., and He, Z. (2015). SOCS3: a common target for neuronal protection and axon regeneration after spinal cord injury. Exp. Neurol. 263, 364-367. doi: 10.1016/j.expneurol.2014.10.024

Liu, Y., Liu, F., Grundke-Iqbal, I., and Iqbal, K. (2011). Deficient brain insulin signalling pathway in Alzheimer's disease and diabetes. J. Pathol. 225, 54-62. doi: 10.1002/path.2912

Ma, Q. L., Yang, F., Rosario, E. R., and Ubeda, O. J. (2009). Beta-amyloid oligomers induce phosphorylation of tau and inactivation of insulin receptor substrate via c-Jun $\mathrm{N}$-terminal kinase signaling: suppression by omega-3 fatty acids and curcumin. J. Neurosci. 29, 9078-9089. doi: 10.1523/JNEUROSCI.1071-09.2009

Mayans, L. (2015). Metabolic syndrome: insulin resistance and prediabetes. FP Essent. 435, 11-16.

Miao, T., Wu, D., Zhang, Y., and Bo, X. (2006). Suppressor of cytokine signaling-3 suppresses the ability of activated signal transducer and activator of transcription-3 to stimulate neurite growth in rat primary sensory neurons. J. Neurosci. 26, 9512-9519. doi: 10.1523/JNEUROSCI.2160-06.2006

Mikulca, J. A., Nguyen, V., Gajdosik, D. A., and Teklu, S. G. (2014). Potential novel targets for Alzheimer pharmacotherapy: II. Update on secretase inhibitors and related approaches. J. Clin. Pharm. Ther. 39, 25-37. doi: 10.1111/jcpt.12112

Morris, J. K., and Burns, J. M. (2012). Insulin: an emerging treatment for Alzheimer's disease dementia? Curr. Neurol. Neurosci. Rep. 12, 520-527. doi: 10.1007/s11910-012-0297-0

Mosconi, L. (2005). Brain glucose metabolism in the early and specific diagnosis of Alzheimer's disease. FDG-PET studies in MCI and AD. Eur. J. Nucl. Med. Mol. Imaging 32, 486-510. doi: 10.1007/s00259-005-1762-7

Mosconi, L., Pupi, A., and De Leon, M. J. (2008). Brain glucose hypometabolism and oxidative stress in preclinical Alzheimer's disease. Ann. N. Y. Acad. Sci. 1147, 180-195. doi: 10.1196/annals. 1427.007

Mullins, R. J., Diehl, T. C., Chia, C. W., and Kapogiannis, D. (2017). Insulin resistance as a link between amyloid-beta and tau pathologies in Alzheimer's disease. Front. Aging Neurosci. 9:118. doi: 10.3389/fnagi.2017.00118 doi: 10.3389/fnagi.2017.00118

Naka, T., Narazaki, M., Hirata, M., and Matsumoto, T. (1997). Structure and function of a new STAT-induced STAT inhibitor. Nature 387, 924-929. doi: $10.1038 / 43219$

Ou, Z., Kong, X., Sun, X., and He, X. (2017). Metformin treatment prevents amyloid plaque deposition and memory impairment in APP/PS1 mice. Brain Behav. Immun. 69, 351-363. doi: 10.1016/j.bbi.2017.12.009

Polizzotto, M. N., Bartlett, P. F., and Turnley, A. M. (2000). Expression of "suppressor of cytokine signalling" (SOCS) genes in the developing and adult 
mouse nervous system. J. Comp. Neurol. 423, 348-358. doi: 10.1002/10969861(20000724)423:2<348::AID-CNE11>3.0.CO;2-W

Qin, H., Niyongere, S. A., Lee, S. J., and Baker, B. J. (2008). Expression and functional significance of SOCS-1 and SOCS-3 in astrocytes. J. Immunol. 181, 3167-3176. doi: 10.4049/jimmunol.181.5.3167

Reger, M. A., Watson, G. S., Green, P. S., and Baker, L. D. (2008a). Intranasal insulin administration dose-dependently modulates verbal memory and plasma amyloid-beta in memory-impaired older adults. J. Alzheimers Dis. 13, 323-331. doi: 10.3233/JAD-2008-13309

Reger, M. A., Watson, G. S., Green, P. S., and Wilkinson, C. W. (2008b). Intranasal insulin improves cognition and modulates beta-amyloid in early AD. Neurology 70, 440-448. doi: 10.1212/01.WNL.0000265401.62434.36

Rui, L., Yuan, M., Frantz, D., and Shoelson, S. (2002). SOCS-1 and SOCS-3 block insulin signaling by ubiquitin-mediated degradation of IRS1 and IRS2. J. Biol. Chem. 277, 42394-42398. doi: 10.1074/jbc.C200444200

Sachithanandan, N., Fam, B. C., Fynch, S., and Dzamko, N. (2010). Liver-specific suppressor of cytokine signaling-3 deletion in mice enhances hepatic insulin sensitivity and lipogenesis resulting in fatty liver and obesity. Hepatology 52, 1632-1642. doi: 10.1002/hep.23861

Smith, P. D., Sun, F., Park, K. K., and Cai, B. (2009). SOCS3 deletion promotes optic nerve regeneration in vivo. Neuron 64, 617-623. doi: 10.1016/j.neuron.2009.11. 021

Son, S. M., Shin, H. J., Byun, J., and Kook, S. Y. (2016). Metformin facilitates amyloid-beta generation by beta- and gamma-secretases via autophagy activation. J. Alzheimers Dis. 51, 1197-1208. doi: 10.3233/JAD-151200

Son, S. M., Song, H., Byun, J., and Park, K. S. (2012). Accumulation of autophagosomes contributes to enhanced amyloidogenic APP processing under insulin-resistant conditions. Autophagy 8, 1842-1844. doi: 10.4161/auto.21861

Soumaya, K. (2012). Molecular mechanisms of insulin resistance in diabetes. $A d v$. Exp. Med. Biol. 771, 240-251.

Starr, R., Willson, T. A., Viney, E. M., and Murray, L. J. (1997). A family of cytokine-inducible inhibitors of signalling. Nature 387, 917-921. doi: 10.1038/ 43206

Steen, E., Terry, B. M., Rivera, E. J., and Cannon, J. L. (2005). Impaired insulin and insulin-like growth factor expression and signaling mechanisms in Alzheimer's disease-is this type 3 diabetes? J. Alzheimers Dis. 7, 63-80. doi: 10.3233/JAD2005-7107

Steffensen, M. A., Fenger, C., Christensen, J. E., and Jorgensen, C. K. (2014). Suppressors of cytokine signaling 1 and 3 are upregulated in brain resident cells in response to virus-induced inflammation of the central nervous system via at least two distinctive pathways. J. Virol. 88, 14090-14104. doi: 10.1128/JVI. 01346- 14

Stein, M. S., Scherer, S. C., Ladd, K. S., and Harrison, L. C. (2011). A randomized controlled trial of high-dose vitamin D2 followed by intranasal insulin in Alzheimer's disease. J. Alzheimers Dis. 26, 477-484. doi: 10.3233/JAD-2011110149

Takeda, S., Sato, N., Uchio-Yamada, K., and Sawada, K. (2010). Diabetesaccelerated memory dysfunction via cerebrovascular inflammation and Abeta deposition in an Alzheimer mouse model with diabetes. Proc. Natl. Acad. Sci. U.S.A. 107, 7036-7041. doi: 10.1073/pnas.1000645107

Talbot, K., Wang, H. Y., Kazi, H., and Han, L. Y. (2012). Demonstrated brain insulin resistance in Alzheimer's disease patients is associated with IGF-1 resistance, IRS-1 dysregulation, and cognitive decline. J. Clin. Invest. 122, 1316-1338. doi: 10.1172/JCI59903

Torisu, T., Sato, N., Yoshiga, D., and Kobayashi, T. (2007). The dual function of hepatic SOCS3 in insulin resistance in vivo. Genes Cells 12, 143-154. doi: 10.1111/j.1365-2443.2007.01044.x

Ueki, K., Kondo, T., and Kahn, C. R. (2004a). Suppressor of cytokine signaling 1 (SOCS-1) and SOCS-3 cause insulin resistance through inhibition of tyrosine phosphorylation of insulin receptor substrate proteins by discrete mechanisms. Mol. Cell. Biol. 24, 5434-5446. doi: 10.1128/MCB.24.12.5434-5446.2004

Ueki, K., Kondo, T., Tseng, Y. H., and Kahn, C. R. (2004b). Central role of suppressors of cytokine signaling proteins in hepatic steatosis, insulin resistance, and the metabolic syndrome in the mouse. Proc. Natl. Acad. Sci. U.S.A. 101, 10422-10427. doi: 10.1073/pnas.0402511101

Uysal, K. T., Wiesbrock, S. M., Marino, M. W., and Hotamisligil, G. S. (1997). Protection from obesity-induced insulin resistance in mice lacking TNF-alpha function. Nature 389, 610-614. doi: 10.1038/39335

van der Heide, L. P., Ramakers, G. M., and Smidt, M. P. (2006). Insulin signaling in the central nervous system: learning to survive. Prog. Neurobiol. 79, 205-221. doi: 10.1016/j.pneurobio.2006.06.003

Vieira, M. N. N., Lima-Filho, R. A. S., and De Felice, F. G. (2017). Connecting Alzheimer's disease to diabetes: underlying mechanisms and potential therapeutic targets. Neuropharmacology. doi: 10.1016/j.neuropharm.2017.11. 014 [Epub ahead of print].

Villemagne, V. L., Fodero-Tavoletti, M. T., Masters, C. L., and Rowe, C. C. (2015). Tau imaging: early progress and future directions. Lancet Neurol. 14, 114-124. doi: 10.1016/S1474-4422(14)70252-2

Walker, D. G., Whetzel, A. M., and Lue, L. F. (2015). Expression of suppressor of cytokine signaling genes in human elderly and Alzheimer's disease brains and human microglia. Neuroscience 302, 121-137. doi: 10.1016/j.neuroscience.2014. 09.052

Wan, W., Cao, L., Liu, L., and Zhang, C. (2015). Abeta1-42 oligomer-induced leakage in an in vitro blood-brain barrier model is associated with upregulation of RAGE and metalloproteinases, and down-regulation of tight junction scaffold proteins. J. Neurochem. 134, 382-393. doi: 10.1111/jnc. 13122

Wan, W., Chen, H., and Li, Y. (2014). The potential mechanisms of Abeta-receptor for advanced glycation end-products interaction disrupting tight junctions of the blood-brain barrier in Alzheimer's disease. Int. J. Neurosci. 124, 75-81. doi: 10.3109/00207454.2013.825258

Wan, W., Zhang, C., Danielsen, M., and Li, Q. (2016). EGb761 improves cognitive function and regulates inflammatory responses in the APP/PS1 mouse. Exp. Gerontol. 81, 92-100. doi: 10.1016/j.exger.2016.05.007

Wang, J., and Campbell, I. L. (2002). Cytokine signaling in the brain: putting a SOCS in it? J. Neurosci. Res. 67, 423-427. doi: 10.1002/jnr. 10145

Xu, Y., Wang, D., Luo, Y., and Li, W. (2015). Beta amyloid-induced upregulation of death receptor 6 accelerates the toxic effect of $\mathrm{N}$-terminal fragment of amyloid precursor protein. Neurobiol. Aging 36, 157-168. doi: 10.1016/j.neurobiolaging. 2014.07.027

Yadav, A., Kalita, A., Dhillon, S., and Banerjee, K. (2005). JAK/STAT3 pathway is involved in survival of neurons in response to insulin-like growth factor and negatively regulated by suppressor of cytokine signaling-3. J. Biol. Chem. 280, 31830-31840. doi: 10.1074/jbc.M501316200

Yang, S. J., Xu, C. Q., Wu, J. W., and Yang, G. S. (2010). SOCS3 inhibits insulin signaling in porcine primary adipocytes. Mol. Cell. Biochem. 345, 45-52. doi: 10.1007/s11010-010-0558-7

Yang, Z., Hulver, M., Mcmillan, R. P., and Cai, L. (2012). Regulation of insulin and leptin signaling by muscle suppressor of cytokine signaling 3 (SOCS3). PLoS One 7:e47493. doi: 10.1371/journal.pone.0047493

Yin, Y., Liu, W., and Dai, Y. (2015). SOCS3 and its role in associated diseases. Hum. Immunol. 76, 775-780. doi: 10.1016/j.humimm.2015.09.037

Zhang, F., and Jiang, L. (2015). Neuroinflammation in Alzheimer's disease. Neuropsychiatr. Dis. Treat. 11, 243-256. doi: 10.2147/NDT.S75546

Conflict of Interest Statement: The authors declare that the research was conducted in the absence of any commercial or financial relationships that could be construed as a potential conflict of interest.

Copyright (c) 2018 Cao, Wang and Wan. This is an open-access article distributed under the terms of the Creative Commons Attribution License (CC BY). The use, distribution or reproduction in other forums is permitted, provided the original author(s) and the copyright owner are credited and that the original publication in this journal is cited, in accordance with accepted academic practice. No use, distribution or reproduction is permitted which does not comply with these terms. 